\title{
》SILLY CAUCASIAN GIRL LIKES TO PLAY WITH SAMURAI SWORDS.« \\ Zur Affektpolitik in QUentin Tarantinos KILL BILL ${ }^{1}$
}

\author{
ACHIM GEISENHANSLÜKE
}

\section{Silly Caucasian Girl}

»Silly caucasian girl likes to play with samurai swords $\aleph^{2}$, mit diesem selbstsicheren Spott begegnet O-Ren Ishii der Braut, nachdem sie ihr den Rücken aufgeschlitzt hat. In ihren Worten drückt sich das Wissen um die Überlegenheit der asiatischen Schwertkämpferin über die weiße Widersacherin aus, die in ihr ureigenes Terrain eindringt und sie nach dem Ehrenkodex der Rache mit den eigenen Waffen zu schlagen versucht. Wenige Minuten später ist O-Ren tot - vom Schwert der Braut skalpiert, als befände sich der Zuschauer nicht nur in einem japanischen Schwertfilm, sondern zugleich in einem amerikanischen Western, in dem der ewige Kampf zwischen Gut und Böse wieder auflodert. »That really was a Hattori Hanzo sword $\ll^{3}$, lauten die letzten Worte O-Rens. Sie verweisen auf das vierte und damit vorletzte Kapitel von KILL BILL: VOLUME 1 zurück: »The man from Okinawa«. Der Titel, der für einen eigenen Film stehen könnte, hält die Identität des legendären Schwertschmieds zurück und bestätigt damit die Technik der verzögerten Enthüllung, die das gesamte vierte Kapitel des Films leitet. In einem gekonnten Spiel von Täuschung und Verstellung begegnen sich die amerikanische Touristin und der mediokre Sushi-Koch, der noch unerkannte Meister der Schmiedekunst und die verkleidete Meisterin des Schwertkampfs, um sich nach einem vorsichtigen gegenseitigen Abtasten auf das gemeinsame Ziel -

1 Den Begriff der Affektpolitik entnehme ich der noch unveröffentlichten Habilitationsschrift »Immune Erzähler. Flaubert und die Affektpolitik des modernen Romans « von Martin von Koppenfels, dem an dieser Stelle auch für viele interessante Diskussionen gedankt sei.

2 KILL BILL: VOLUME 1, 01:30:24.

3 KILL BILL: Volume 1, 01:33:17. 
eben: Kill Bill - zu einigen. Mythologische und märchenhafte Elemente verschmelzen in der Sequenz miteinander und reflektieren zugleich auf einen ironischen Tausch der Traditionen, der mit dem kulturwissenschaftlichen Schlagwort >Interkulturalität` nur unzulänglich beschrieben wäre.

»Literatur, Texte, Filme, Medien sind Träger kultureller Darstellung und Kodierung, wie sie für Prozesse des Kulturtransfers entscheidend sind. Durch sie werden Traditionen und Überzeugungssysteme, Schlüsselsymbole- und praktiken sowie Fremd- und Selbstbilder ausgebildet und für die praktische interkulturelle Auseinandersetzung geradezu aufbereitet bzw. hierfür strategisch einsetzbar gemacht. ${ }^{4}$

So fasst Doris Bachmann-Medick das gängige kulturwissenschaftliche Verständnis von Interkulturalität zusammen. Quentin Tarantinos Bild der japanischen und der amerikanischen Kultur in KILL BILL wirkt wie eine Parodie auf die Dialektik von Selbst- und Fremdbild, die den Kulturwissenschaften die Grundlage geben soll. Die Möglichkeit einer ernsthaften Untersuchung von Selbst- und Fremdbildern wird vom Film gleich auf mehreren Ebenen unterlaufen: Die Japanerin, die sich über das >silly caucasian girl lustig macht, ist im Film selbst ein Kind Amerikas, geboren auf einer amerikanischen Militärbasis, und damit lebendige Verkörperung der Hybridität, die den Film kennzeichnet. Darüber hinaus konfrontiert der Schwertkampf im Kontext einer Verschiebung der traditionellen Geschlechterrollen nicht nur zwei Frauen anstelle zweier männlicher Heroen miteinander, von denen die eine darüber hinaus in der Verkleidung des gelben Motorradanzugs auftritt, mit dem Bruce Lee in seinem letzten Film auf der Leinwand erschienen ist. Lesbar ist der Kampf, dramatischer Höhepunkt des ersten Teils, daher auch als differierende Wiederholung des Zweikampfes von Cowboy und Indianer, hier: von Cowgirl und Indianerin, und damit zugleich als zwiespältiger Kommentar zur kulturellen Hegemonie Amerikas, die der erste Teil zu bestätigen scheint und der zweite Teil des Films konsequent unterläuft. Spielt KILl BILl: Volume 2 im Unterschied zum ersten Teil fast ausschließlich im amerikanischen Westen, so geschieht dies nur, um den Mythos Amerika um so gründlicher zu dekonstruieren. Die erhabene Tradition des Schwertkampfes, die in die Schlacht im Haus der blauen Blätter mündet, wird im zweiten Teil außer Kraft gesetzt und durch eine reflexive Ebene abgelöst, die das Pathos des ersten Teils bis zum verstörenden Schluss nicht verstärkt, sondern ironisch aufbricht. Ist das

4 Vgl. Doris Bachmann-Medick (Hg.): Kultur als Text. Die anthropologische Wende in der Literaturwissenschaft, Frankfurt a.M.: Fischer 1996, S. 8. 
Schwert, das der Braut vom Mann aus Okinawa überreicht wird, das zentrale Symbol für den Mythos, der in KILL BILL erzählt und zugleich dekonstruiert wird, so lassen sich an der Geschichte des Schwertes auch die wesentlichen Stationen des Handlungszusammenhangs - nichts anderes hatte Aristoteles unter dem Begriff Mythos verstanden ${ }^{5}$ aufzeigen, der Tarantinos vierten Film kennzeichnet. Die folgenden Überlegungen konzentrieren sich - neben Exkursen zu Homer und der ganz anderen Begegnung von fernöstlicher und amerikanischer Tradition im Kino Takeshi Kitanos und nach grundsätzlichen Überlegungen zur Affektpolitik des Films - zunächst auf das vierte Kapitel des Films, um anhand der Leitmetapher des Schwertes den Remythisierungstendenzen des Films nachzugehen und deren Dekonstruktion aufzuzeigen. ${ }^{6}$

\section{Achills Schwert}

Mit dem Schwert als Symbol für die fast märchenhaft anmutende Unbesiegbarkeit der gelbhaarigen Kriegerin nimmt Quentin Tarantino in KILL BILL eine wirkungsmächtige Metapher aus der Kulturgeschichte auf. Nicht nur die immer wieder verfilmte Geschichte des Königs Artus und seines Schwertes Excalibur spricht für die fetischisierende Kraft, die von Waffen wie dem Schwert ausgeht. Das Erringen einer Waffe, die dem Besitzer besondere Fähigkeiten und Macht verleiht, ist uralter Bestandteil der europäischen Schriftkultur und weist bis zu Homer zurück. Mit dem Schwert tötet Achill Hektor und sichert sich den Ruhm, auf ewige Zeiten der größte Krieger der Achaier zu sein:

"Also rief er und riß das schneidende Schwert aus der Scheide,

Welches herunter ihm hing an der Hüfte, das wuchtige, große.

Vorwärts schoß er wie geduckt, wie ein fliegender Aar aus der Höhe

Nieder zur Ebene stößt durch finstere Wolken, ein zartes

Lämmlein oder den feig sich bergenden Hasen zu greifen. « ${ }^{7}$

5 "Die Nachahmung von Handlung ist der Mythos «, Aristoteles: Poetik, Stuttgart 1982, S. 19.

6 Vgl. in diesem Kontext Peter Körte: »Was im Internet der Hypertext ist als aktive Dekonstruktion von Autorprinzip und gesicherter Grenzen zwischen verschiedenen Textarten, das ließe sich im Gestus des Erzählens, im Umgang seiner Filme mit anderen Filmen durchaus als Dekonstruktion beschreiben. « Peter Körte: "Geheimnisse des Tarantinoversums. Manie, Manierismus und das Quäntchen Quentin - Wege vom Videoladen zum Weltruhm«, in: Robert Fischer/Peter Körte/Georg Seeßlen: Quentin Tarantino, Berlin: Bertz + Fischer 2004, S. 11-64, hier S. 26.

7 Homer: Ilias,: griechisch und deutsch. Übertr. von Hans Rupé, Düsseldorf [u.a.]: Artemis \& Winkler 2001, Zweiundzwanzigster Gesang, Vers 306-310. 
Nicht von ungefähr erscheint die Braut zum Schluss des Films als >Löwin`, die ihr Junges zurück gewonnen hat. Wie Achill, Löwe und Adler zugleich, zeichnet sich die Braut nach einer gewissenhaften Ausbildung durch überragende Kampfkunst und eine besondere Waffe aus. Das Schwert, >das wuchtige, großeく, das Achill an der Hüfte hängt wie ein zweiter Phallus, zwingt den Gegner in den Staub. Von dem Zorn beseelt, von dem die Ilias ein Loblied singt, ist Achill nicht bereit, dem Gegner die gewünschte ehrenvolle Bestattung zu gewähren: »Ach, ich kenne dich wohl und seh' es deutlich, du warest / Nicht zu erweichen; du trägst ein eisernes Herz doch im Busen. ${ }^{8}$ Ein eisernes Herz, durch nichts $\mathrm{zu}$ erweichen, nicht einmal durch den Anblick der eigenen Tochter, ist auch die Braut in KILL BILL. Von der erhabenen Kraft, die von Rache und Zorn ausgehen, erzählt Tarantino wie schon Homer in der Ilias, und der einleitende Titelsong Bang Bang von Nancy Sinatra nimmt das einleitende »Singe mir, o Göttin, vom Zorn des Peleiden «" ironisch auf: Tarantinos Appell an Nancy Sinatra, deren Song damit die Funktion einer Apostrophe, einer Anrufung der Muse, erhält, das >Singe mir, o Göttin, vom Zorn der Braut`, mit dem der Film beginnt, nimmt die Tradition der erhabenen und blutdurchtränkten Rachegeschichten auf, die nicht erst seit Shakespeare die Geschichte der Literatur durchziehen.

Dem Tod Hektors geht in der Ilias eine komplexe Geschichte voraus, die dem Helden Achill erst zu den Waffen verhilft, die seinen Sieg und Ruhm sichern. Als Patrokles in der Rüstung Achills von Hektors Hand fällt, bittet Achills Mutter Thetis den ihr verpflichteten Hephaistos um neue Waffen für den Sohn. Den Schild und die Rüstung, die Hephaistos schmiedet, geben in der Form des Wunderbaren ein getreues Abbild der Welt, »die Erde, das Meer und den Himmel, / Ferner den vollen Mond und die unermüdliche Sonne, / Dann auch die Sterne dazu, die den Himmel umkränzen. ${ }^{10}$ Als vollständiges Abbild der Welt erstrahlen die Waffen in einem Glanze, dem allein Achill standhält: »Doch Achilleus / Faßte, sobald er sie sah, noch stärker der Zorn, und die Augen / Unter den Lidern erstrahlten so schrecklich wie Flammen des Brandes. ${ }^{11}$ Den Impuls der Rache, der Achill nach dem Tod des Patrokles beseelt, entfacht der Anblick der Waffe neu. Das Feuer, aus dem die Waffen geschmiedet sind, scheint in den Helden selbst einzugehen und mit ihm zu verschmelzen. Waffe und Mensch, Fleisch und Stahl, werden zu einer Einheit, die der Krieger Achill verkörpert, der nicht nur den Tod Hektors verursacht, sondern damit auch den eigenen in Kauf zu nehmen bereit ist. Mit den gottgegebenen Waffen erfüllt sich das selbstgewählte Schicksal

8 Ebd., Vers 356-357.

9 Ebd., Erster Gesang, Vers 1.

10 Ebd., Dreiundzwanzigster Gesang, Vers 483-485.

11 Ebd., neunzehnter Gesang, Vers 15-17. 
Achills, ruhmvoll und jung sterben zu müssen. `Live fast, die young`: Die Bereitschaft, den eigenen Tod als Preis für die Rache zu akzeptieren, bestätigt den erhabenen Charakter, für den Achill wie kein anderer Held der Antike einsteht und für den er von Hölderlin, »so stark und zart, die gelungenste, und vergänglichste Blüthe der Heroenwelt $\aleph^{12}$, so geliebt wurde.

\section{Schmerz}

Stark und zart, wie Hölderlins verliebtes Auge Achill sah, ist auch die Rachegöttin, von der Tarantinos Film erzählt. Nicht nur der erhabene Affekt des Zorns, nach Aristoteles »ein mit Schmerz verbundenes Trachten nach dem, was uns als Rache für das erscheint, worin wir eine Kränkung unserer selbst oder eines der unsrigen erblicken von jemandem, dem das Kränken nicht zukommt « $^{13}$, verbindet die Braut mit dem antiken Vorbild Achills: »man ist nämlich zornig, wenn einem Schmerz zugefügt wird « ${ }^{14}$, setzt Aristoteles hinzu. Lesbar wird die Rache als Äquivalent des Schmerzes, den jemand erlitten hat. Tarantinos Film ist daher nicht allein eine Geschichte der Rache, sondern zugleich eine des Schmerzes, eine Passionsgeschichte, die von starken Affekten wie Zorn, Scham und Liebe erzählt. Ausgespart wird allein der Affekt, der in der Moderne, bei Freud wie bei Heidegger, die meiste Aufmerksamkeit gefunden hat: die Angst. Das Überspringen der Angst liegt in der Struktur des Zorns begründet: Als Aussicht auf die zukünftige Wunschverwirklichung der Rache schließt Zorn die Angst aus. Angst könnte allein das Nichterreichen des Ziels betreffen, auf das der Zorn hinarbeitet. Genau das aber setzt der Zorn voraus: dass das Ziel erreichbar ist. Leitet Aristoteles in der Rhetorik Affekte grundsätzlich unter der Differenz von Lust und Unlust ein, so ordnet er den Zorn, dessen Struktur er der Ilias abliest, im Unterschied zur negativen Besetzung der Angst eindeutig der Lust zu, weil er das Erreichen des gewünschten Ziels voraussetzt. Aristoteles stellt fest, »dass der Zorn in jedem Fall von einem gewissen Lustgefühl begleitet ist, das auf der Hoffnung, sich rächen zu können, basiert; denn es ist angenehm, sich vorzustellen, man werde das, wonach man strebt, erreichen. Niemand aber strebt nach dem, was ihm

12 Friedrich Hölderlin: Sämtliche Werke, Briefe und Dokumente. Hrsg. von D. E. Sattler, Bremer Ausgabe, Band VI. 1797-1799, München: Luchterhand 2004, S. 229.

13 Aristoteles: Rhetorik. Übersetzt, mit einer Bibliographie, Erläuterungen und einem Nachwort von Franz G. Sieveke, München: Fink 1980, S. 85.

14 Ebd., S. 87. 
unerreichbar erscheint. ${ }^{15}$ Das Gelingen der Rache steht in KILL BILL außer Frage, und die Lust, die der Zuschauer aus dem Spektakel gewinnt, rührt aus der unmittelbaren Identifikation mit dem Ziel der Braut, das nicht nur möglich, sondern zugleich notwendig und wirklich erscheint.

Die innere Notwendigkeit der Rachegeschichte verweist auf die dramatische Tradition und damit auf den für die Tragödie zentralen Begriff des Pathos. Mit dem Schmerz steht das Pathos des Films auf dem Spiel. »Pathos bezeichnet den Bereich des Ästhetischen, der traditionell die Vermittlung des Schmerzes zu leisten hatte: das Feld der negativen Affekte, sofern sie ästhetischer Bearbeitung zugänglich sind. ${ }^{16}$ Wie Martin von Koppenfels darlegt, besteht die Funktion des Pathos in der Übersetzung von Schmerz in Affekte. Nichts anderes leistete die attische Tragödie in der Erregung des >eleos $\prec$, das in seiner Unübersetzbarkeit zwischen Mitleid und Jammern davon zeugt, dass es seinen Grund in einer Form der Übertragung findet, die zunächst die Verwandlung von Schmerz in Affekte und dann die von Affekten in Empathie leistet. Niemand will leiden. Dennoch geht eine starke identifikatorische Kraft von den Leiden der tragischen Helden aus, deren Gegenstand eben nicht der Schmerz, sondern der aus der Übertragung hervorgegangene Affekt ist - im Falle der Braut der gewaltige Zorn, der den Film bald mit einer Unzahl von Leichen bevölkert. Vor diesem Hintergrund lohnt es sich, nicht allein nach der Rache, sondern auch nach dem Schmerz zu fragen, der den Zorn erst begründet.

Schmerz und die damit verbundene Bewegung der »Erstarrung, Versteinerung ${ }^{17}{ }^{17}$, von der die mythische Mutterfigur Niobe bei Sophokles und Hölderlin sprachlos Zeugnis ablegt, betrifft die Protagonistin von Tarantinos Film gleich auf mehrerlei Weise: als körperlicher Schmerz, der ihr von dem Deadly Viper Assassination Squad unter Leitung von Bill zugefügt wird, als Schmerz durch die Vergewaltigungen, deren nunmehr wirklich erstarrtes, komatöses Opfer sie im Krankenhaus wird, als demütigender Schmerz in der grausamen Lehrzeit unter Pai Mei, und schließlich als gleichermaßen körperlicher wie seelischer Schmerz, den der Verlust des Kindes für sie bedeutet. Angesichts der enormen Schmerzerfahrungen, die sie hinter sich hat, hat die Braut nichts mehr zu verlieren - bis zu dem Moment, als sie ihrer tot geglaubten Tochter in die Augen sieht und im Spiel im Spiel tot umfällt. In der Begrifflichkeit der Tragödie, deren Logik Tarantinos Rachegeschichte immer wieder bemüht, markiert das Wiedersehen daher den tragischen

15 Ebd., S. 85.

16 Martin von Koppenfels: "Schmerz. Lessing, Duras und die Grenzen der Empathie«, in: Robert Stockhammer: Grenzwerte des Ästhetischen, Frankfurt a.M.: Suhrkamp. 2002, S. 118-145, hier S. 122 f.

17 Ebd., S. 144. 
Zusammenhang von Peripetie und Wiedererkennung, den Aristoteles in der Poetik festlegt: »Die Peripetie ist, wie schon gesagt wurde, der Umschlag dessen, was erreicht werden soll, in das Gegenteil, und zwar, wie wir soeben sagten, gemäß der Wahrscheinlichkeit oder mit Notwendigkeit. « ${ }^{18}$ Ähnliches gilt für die Wiedererkennung:

»Die Wiedererkennung ist, wie schon die Bezeichnung andeutet, ein Umschlag von Unkenntnis in Kenntnis mit der Folge, dass Freundschaft oder Feindschaft eintritt, je nachdem die Beteiligten zu Glück oder Unglück bestimmt sind. Am besten ist die Wiedererkennung, wenn sie zugleich mit der Peripetie eintritt. ${ }^{19}$

In KILL BILL scheinen Wiedererkennung und Peripetie zwar zusammen zu fallen. Die Konfrontation mit der eigenen Tochter, die Bill sorgfältig inszeniert, bewirkt jedoch keinen Umschlag des Ziels, das erreicht werden soll: Der Braut geht es weiterhin darum, Bill zu töten, und dieses Ziel verwirklicht sie in relativ kurzer Zeit. Insofern verschiebt der Umschlag von Unkenntnis in Kenntnis, der die Wiedererkennung ausmacht, nur das Ziel der Braut nach der Befriedigung der Rache, nach der sie strebt. Das doppelte Ziel der buchstäblichen Verdoppelung von Beatrix Kiddo in das filmgeschichtlich relevante Kürzel B. B. ist die erhabene Aussicht auf Rache und die eher untragische Aussicht eines Zusammenlebens mit der tot geglaubten Tochter. Für die Affektpolitik des Films heißt das aber: Schmerz und Zorn aus enttäuschter Liebe werden durch Liebe (und damit die Möglichkeit zu neuen Schmerzen) abgelöst. Der Film befindet sich daher in dem Dilemma, den Affekt, der den Handlungszusammenhang des Films bis zum Schluss bestimmte, abschließend außer Kraft setzen zu müssen. Das gelingt nur in der Wiedereinsetzung des Mythos der mütterlichen Liebe, der das eigentlich zu erwartende tragische Ende, den Tod, ersetzt und damit zugleich einen Umschlag von der Tragödie in die Komödie andeutet, den der Film mit allen Mitteln zu vermeiden sucht. Komisch im dramatischen Sinne ist der Schluss des Films allein deshalb nicht, weil das Ende mit dem Mythos der mütterlichen Liebe pathetisch neu besetzt wird. Die Unwahrscheinlichkeit von Liebe jenseits des Kitsches glaubhaft zu machen, scheint daher eines der Ziele des Films zu sein.

Für die Unwahrscheinlichkeit, die Tarantinos Film beseelt, hat Niklas Luhmann die passende Formel gefunden. In Liebe als Passion bezeichnet er Liebe als »eine ganz normale Unwahrscheinlichkeit « ${ }^{20}$, als eine Stei-

18 Aristoteles: Poetik, S. 35.

19 Ebd.

20 Niklas Luhmann: Liebe als Passion. Zur Codierung von Intimität, Frankurt a.M.: Suhrkamp 1982, S. 10. 
gerung der Wahrscheinlichkeit von Unwahrscheinlichkeit, die schon Aristoles mit dem sophistischen Ausspruch bekräftigt hatte, »es ist wahrscheinlich, dass sich vieles gegen die Wahrscheinlichkeit abspielt. « ${ }^{21}$ Neben dem Schmerz erscheint der Affekt der Liebe somit als zweites Movens der Rachegeschichte, die der Film entfaltet, indem er beide zunächst voneinander trennt, um sie zum Schluss wieder zusammen zu führen: Auf die Liebe von Bill und der Braut, die in Bills unpathetischen Worten mit dem Braten in der Röhre endet, folgt der Schmerz, den Bill Kiddo zufügt, nachdem er den Schmerz nicht verwinden konnte, dass sie nicht tot ist, sondern ihn nur ganz profan verlassen hat. In der aus der Perspektive der leidenden Heldin ganz und gar unwahrscheinlichen Begegnung mit der eigenen Tochter fallen Liebe und Schmerz dann wieder zusammen - ein Happy end der besonderen Art, auf das der Tod Bills nur noch beiläufig folgt. Was sich in den aus Schmerz und Freude gemischten Tränen der Braut, die zum Schluss des Films nun wirklich zur Mutter geworden ist, zeigt, ist der kathartische Anfall, den Aristoles auf den Zuschauer verschoben hatte: die Lösung aus der Erstarrung, die der Wunsch nach Rache bedeutete. Die tragische >katharsis` als Waffe gegen die Waffengewalt der wutentbrannten Braut aufzubieten, gibt dem Film eine verstörende Wendung, indem sie die vom Weinen ins Lachen kippende Braut in eine ganz normale Hysterikerin verwandelt. Der Anfang der kathartischen Kur, an deren Ende Freud die nüchterne Forderung stellt, »hysterisches Elend in gemeines Unglück zu verwandeln $\ll^{22}$, steht an ihrem Ende. Im erhabenen Affekt des Zorns zu verharren, ist der Braut so wenig gegeben wie Achill, den die Trauer um den Freund und der Zorn auf Hektor wieder in den Kampf schickt, der mit seinem Tod endet. Nicht sterben zu müssen, nicht im Affekt des Zorns heimisch bleiben zu können, bedeutet daher die Notwendigkeit, neue Übertragungen des Schmerzes einzugehen, eben: neu zu lieben. Dass Liebe und Schmerz eins und daher beide der Übertragung, also filmfähig sind, ist einer der Schlüsse, die sich aus KILL BILL ziehen lassen. Was für den Film entscheidend ist, sind jedoch Fragen der Darstellung: Die Geschichte des Schmerzes muss anders erzählt werden als die der Liebe, weil beiden unterschiedliche Übertragungstechniken zugrunde liegen. Die - wenn auch erzähl-technisch anders umgesetzte - Linearität der Rachegeschichte, die die Todesliste der Braut vor Augen führt, wird durch den Einbruch des Unwahrscheinlichen, das Liebe heißt, gebrochen. Insofern dient der Bruch mit einer linearen Erzählweise, der schon PULP FICTION berühmt machte, nur der stärkeren Motivierung des Handlungs-

21 Aristoteles: Poetik, S. 59.

22 Sigmund Freud: Studien zur Hysterie, in: Gesammelte Werke I, Frankfurt a.M.: Fischer 1999, S. 312. 
zusammenhangs, den Aristoteles Mythos nannte und den er den Gesetzen von Peripetie und Wiedererkennung unterstellte. Zeigt der Film einen doppelten Umschlag, den von Liebe in Schmerz und den von Schmerz in Liebe, so verhalten sich beide zum Mythos chiastisch: Der Umschlag von Liebe in Schmerz führt zu einer linearen Verlaufs-geschichte, an deren Ende allein der Tod stehen kann. Der Bruch, den der Schluss des Films als unwahrscheinliche Form des Umschlags von Schmerz in Liebe zeigt, verläuft nicht linear, sondern unkontrollierbar, ereignishaft -, eben so, wie sich starke Affekte verhalten, wenn sie noch nicht rationalisiert sind. Rationalisiert und in eine operationale Form der Logik gezwungen ist daher zwar die Rachegeschichte des Films. Was Tarantino in KILL BILL unternimmt - und das verweist trotz der ständigen Präsenz von Gewalt viel eher als auf RESERVOIR Dogs und PULP FICITION auf den bis heute von Publikum und Kritik unterschätzten JACKIE BROWN -, ist, wie auch in vergleichbaren asiatischen Filmen wie TIGER \& DRAGON oder THE HOUSE OF THE FLYING DAGGERS, jedoch der Versuch, in der Geschichte der Gewalt eine Geschichte der Liebe zu erzählen. Liebe und Gewalt, die im gesamten Verlauf des Films eng aneinander gebunden, ja fast miteinander deckungsgleich sind, wieder zu trennen, erscheint als der utopische Rest, der in KILL BILL zum Schluss verhandelt wird. Das Schwert, das den Rachefeldzug begleitet, verkörpert daher zunächst die Gewalt, die dem Film seine innere Logik gibt und die erst zum Schluss durch das Ereignis der mütterlichen Liebe aufgehoben wird - anders als in Kleists Penthesilea, die ihre Waffen nur abgibt, um sich aus Jammer und Reue einen Dolch zu formen, der ihr den ersehnten Tod bringt. Nicht nur das tragische Ende unterscheidet Kleists gewalt- wie liebesbereite Heldin von der Tarantinos. Das Schwert, das ihr die ersehnte Rache bringen soll, formt die Braut nicht selbst. Dafür braucht sie den Mann aus Okinawa.

\section{The Man from Okinawa}

Als Beatrix Kiddo, wie die Braut im zweiten Teil des Films heißen darf, von den Kugeln ihrer Tochter getroffen wird und theatralisch auf den Boden sinkt, versichert sich das zweifelnde Kind, das alles sei doch nur ein Spiel. Ironisch, als Spiel im Spiel, nimmt der Film die Logik auf, die bereits den ersten Teil bestimmte: den Nimbus der Unbesiegbarkeit, den die Braut durch das Schwert erlangt, das ihr am Ende des vierten Kapitels feierlich überreicht wird, um ihr im »Showdown at the House of Blue Leaves« umgehend zum Sieg zu verhelfen. Nicht durch die Hand der Braut, durch das Schwert, dem ihre letzten Worte gelten, scheint O-Ren zu fallen. Kaum fassbar erscheint ihr zunächst, dass die Braut mit 
einem Hattori Hanzo Schwert gegen sie antritt: »You lie $\aleph^{23}$, schleudert sie ihrer Widersacherin entgegen, bis das aus der Scheide gezogene Schwert sie eines besseren belehrt. Insofern erfüllt das vierte Kapitel des Films, das die Geschichte des Mannes von Okinawa erzählt, die Funktion eines retardierenden Moments innerhalb der blutigen Rachegeschichte und zugleich die einer Begründung des Mythos, der sich um Hattori Hanzos Schwert und Bill rankt, ohne jemals als solcher erzählt zu werden.

Am Beginn der Begegnung des Schmiedes und der Kämpferin steht die Travestie kultureller Muster Japans und Amerikas: Als lächelnde amerikanische Touristin verkleidet, ausgestattet mit einem dürftigen Wortschatz, der das Gespräch zu einem Spiel der Konventionen werden lässt, betritt die Braut das Geschäft Hattori Hanzos.

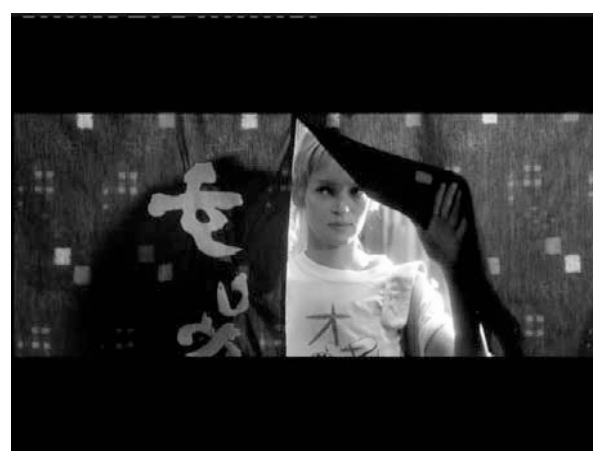

\section{Abb. 1: Beatrix Kiddo als Touristin}

Neben die Darstellung der konventionellen Kraft der Sprache - Begrüßungs- und Dankesworte sind alles, was die Braut zunächst ins Spiel einbringt - tritt die Komik, die in der Figur des Gehilfen auftritt, der sich dem Auftrag seines Chefs zu entziehen sucht, weil er seine ssoap operas< schaut. »For 30 years, you make the fish, I get the sake. $\aleph^{24}$ Die Szene markiert daher nicht nur »a parodic decline in Hanzo's fortune $\ll^{25}$, wie D.K. Holm in An Unofficial Casebook zu KILL BILL meint, sondern zugleich eine Verschmelzung von amerikanischen Slapstick und japanischer Serienproduktion. Auf der japanischen Insel trifft die Braut auf sehr amerikanische Fernsehgewohnheiten, und das in einem Ambiente, das nicht nur durch die der TV-Serie SHADOW WARRIORS entlehnte Figur des Hattori Hanzo, sondern auch durch das auf einen Raum beschränkte

23 KILL BILL: VOLUME 1, 01:27:15.

24 KILL BILL: VOLUME 1, 00:48:33.

25 D.K. Holm: Kill Bill. An Unofficial Casebook, London: Glitterbooks 2004, S. 55. 
Dekor an eine Fernsehserie erinnert. Mit dem lächelnden Auftritt der Braut als Touristin ruft Tarantino im Zitat des Zitats zugleich das Genre des Detektivfilms ab, demzufolge ein Geheimnis nur ergründet werden kann, wenn sich der Held verkleidet in die Höhle des Löwen wagt, um Informationen zu bekommen, die ihm auf seinem weiteren Weg hilfreich sein können.

Die Strategie der Verstellung, die der Szene zugrunde liegt, geht daher mit der Instanz des Geheimnisses einher. Dass in diesem Spiel nicht nur die Braut betrügt, sondern auch der Koch, der seine Identität hinter der Maske der Mittelmäßigkeit zu verbergen sucht, erfährt der Zuschauer im Laufe des Gesprächs, ohne allerdings das Geheimnis um Hattori Hanzo und Bill lüften zu können. Alles, was der Film preisgibt, ist die Tatsache, dass vor 28 Jahren etwas vorgefallen ist, das Hanzo und die Braut zu Verbündeten im Kampf gegen Bill macht. Das Zurückhalten von Fähigkeiten und Informationen, das die Episode bestimmt, vollzieht der Film selbst mit, um aus dem legendären Schmied eine Legende zu schmieden, die in der Fertigung seines letzten Schwertes ihren Höhepunkt finden soll.

Im Verhältnis zum von Verstellung und Komik bestimmten Beginn ist das vierte Kapitel durch den Umschlag des Komischen ins Erhabene gekennzeichnet, der Tarantinos Kunst der Travestie schon in PULP FICTION bestimmte. Den Umschlag vollzieht die Macht der Sprache, sobald sie den Rahmen der Konvention verlässt. Auf der Grenze zwischen Konvention und Intimität, die der Touristin im Geschäft verwehrt bleibt, steht der Name. Auf die Frage, wen sie in Okinawa besuche, antwortet die Braut schlicht mit: »Hattori Hanzo. ${ }^{26}$ Der Name fungiert als Scharnier im dramatischen Geschehen. Er markiert den Umschlag der Sprache - fortan ist das Gespräch durch eine beständige Form des Wechselns vom Japanischen ins Englische und umgekehrt bestimmt - wie die Offenbarung der Identität, die der Koch zwar zu verbergen sucht, die sich dem Zuschauer und der Braut aber schon öffnet, als er im Ärger über seinen Gehilfen das Messer virtuos auf den Block wirft. Hinter der Fassade des heruntergekommenen Geschäfts, das Hattori Hanzo betreibt, öffnet sich eine eigene Geschichte, die in KILL BILL nur erzählt wird, insofern sie auf die Geschichte der Braut bezogen ist.

$\mathrm{Zu}$ der Intimität, die das Aussprechen des Namens errichtet, zählt der Schauplatzwechsel. Im Dachboden, der wie in Kafkas Proce $\beta$-Roman einer Rumpelkammer des Unbewussten gleicht, trifft die Braut nicht auf das eine Schwert, das ihr zugedacht ist, sondern auf einen unbezahlbaren Schatz von Schwertern, der auf Hanzos ruhmreiche Vergangenheit verweist. Dass er die Schwerter allein aus ästhetischen und sentimentalen

26 KILL BILL: Volume 1, 00:47:42. 
Gründen behält, wie Hanzo der Braut versichert, zeugt von seinen Anstrengungen, die erhabene Vergangenheit als Schmied ästhetisch transformiert zu bewahren - Grundlage für die spätere Übereinkunft, ein Schwert für die Braut zu verfertigen. Die Wiederaufnahme des Spiels der Konventionen - "you like samurai swords, I like baseball $\aleph^{27}$ - dient nur der Probe der Heldin, die den ihr zugeworfenen Baseball mit einem Schlag in zwei Hälften schlägt. Die mythische Probe stattet die Braut mit dem Fetisch der Waffe aus, um ihre Identität im Glanz des Schwertes neu zu begründen: Anders als im Kampf mit den 88 Yakuzas dient der Blick, den die Braut auf das Schwert wirft, nicht nur der Abwehr der anderen, sondern zugleich der Versicherung des eigenen Selbst: Im Spiegel der Klinge erblickt sich die Braut als Spiegelbild der Klinge, um jene Ganzheit des Selbst zu erlangen, die schon Jacques Lacan in seinen Überlegungen zum Spiegelstadium als eine kindliche Form der Selbsttäuschung ausgegeben hat. ${ }^{28}$ Der Zauber, der wie im Märchen von der Macht des Schwertes ausgeht, erweist sich in seiner mythisierenden Kraft zugleich als eine Form der Täuschung, der die Heldin auf ihrem Rachefeldzug unterliegt: An die Stelle der dissimulatio, der täuschenden Verbergung vor anderen ${ }^{29}$, tritt die Selbsttäuschung, die die durch das Schwert begründete Ordnung der Gewalt zugleich in den Bereich der Hybris verweist.

Ausdruck dieser Hybris ist der Verstoß Hattori Hanzos gegen den vor 28 Jahren getätigten Schwur, keine »instruments of death $\aleph^{30}$ mehr herzustellen. Auf den komischen Beginn der Szene folgt das tragische Ende, das zugleich den erhabenen Charakter der Schwertüberreichung unterstreicht, verstärkt - und zugleich ironisch gebrochen - durch Gheorge Zamfirs Flötengesang The Lonely Sheperd. ${ }^{31}$ Den Umschlag in das selbst wiederum der Parodie würdige Genre des Erhabenen vollziehen alle beteiligten Figuren gleichermaßen: Hattori Hanzo, der vom Koch in die Rolle des Schwertschmiedes zurückgefunden hat, sein Gehilfe, der ihm nun willig assistiert, und schließlich die Braut, die, nun im traditionellen japanischen Gewand gekleidet, auf die Überreichung

27 KILL BiLL: Volume 1, 00:50:52.

28 Vgl. Jacques Lacan: »Le stade du miroir comme formateur de la fonction du Je«, in: Ecrits, Paris: Seuil 1966, S. 93-100.

29 Vgl. Alexander Kosěnina: „Verstellung «, in: Historisches Wörterbuch der Philosophie, hrsg. von Joachim Ritter, Karlfried Gründer und Gottfried Gabriel. Band 11: U-V, Darmstadt: Wissenschaftliche Buchgesellschaft 2001, S. 938-942, hier S. 938.

30 KILL BILL: VolUMe 1, 00:55:00.

31 Zu Musik in KILL BILL vgl. Georg Seeßlen, Zärtliche Zerstörungen. Anmerkung zur Musik in Tarantinos Filmen, Berlin: Bertz + Fischer 2004 (film: 1), S. 6586. 
des Schwertes das gleiche Wort folgen lässt, mit dem die Konversation scheinbar unschuldig begann: »Domo. $\ll^{32}$

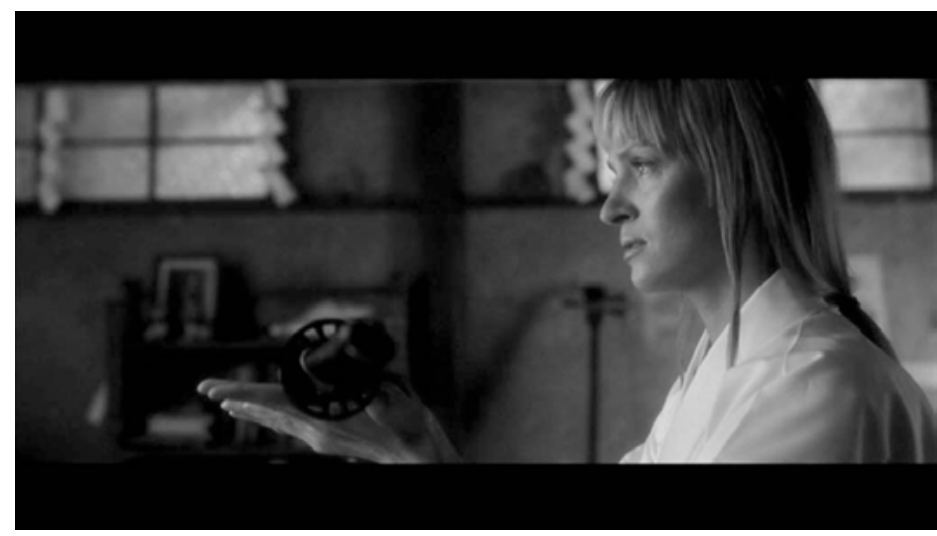

Abb. 2: Beatrix Kiddo bei der Überreichung des Schwertes

Vor diesem Hintergrund unterstreicht der magische Spruch Hanzos bei der Übergabe des Schwertes in Anspielung auf den Samourai Film Makai tenshô von Kinji Fukusaku aus dem Jahre 1981 nicht nur das Gelingen der ihm gestellten Aufgabe, sondern zugleich jenes Moment der Hybris, ohne das die Tragödie nicht auskommen kann: »If, on your journey, you should encounter god, god will be cut. $\ll^{33}$ Auslöser der Rachgeschichte war das Moment der Übertretung, das das zur Legende gewordene >Massacre at Two Pines` in Gottes eigenen Räumen markierte. Auch die Rache, die Hanzo und die Braut verbindet, erscheint als ein Moment der Übertretung, als Überschreiten eines Maßes, das durch Hanzos Schwur ebenso markiert wird wie durch das anschließende Gemetzel im Haus der blauen Blätter. »I overreacted « ${ }^{34}$, lautet die eben so schlichte wie hilflose Antwort Bills auf die Frage, die ihm Kiddo zum Schluss des Films stellt, warum er ihr eine Kugel in den Kopf habe jagen müssen. Das Prinzip der Überreaktion, das Bill entschuldigend anführt, gilt nicht nur für ihn, sondern ebenso für die rachedurstige Braut, schließlich für den ganzen Film, der in seiner Überfülle an filmischen Verweisen selbst nichts anderes als beständige Form der >overreaction< ist, der überreizten Reaktion auf die Reize, die andere Filme bedeuten Filme wie die Kitanos, die sich gerade im Blick auf das ästhetische

32 KILL BILL: VOLUME 1, 00:44:00.

33 KILL BILL: VolUME 1, 00:54:50.

34 KILL BILL: Volume 2, 01:52:09. 
Prinzip der Überreaktion allerdings signifikant von Quentin Tarantinos Kino unterscheiden.

\section{Brüder}

Takeshi Kitanos ZATOICHI nimmt die in unzähligen Variationen erzählte Legende des einsamen Schwertkämpfers auf, dessen weibliche Variante Tarantino in KILL BILL vorführt. Allerdings mit einem gewichtigen Unterschied: Die lineare Folgerichtigkeit, mit deren Hilfe Gewalt in KILL BILL dargestellt wird, setzt Kitano außer Kraft. In seinen Filmen erscheint Gewalt in der Weise, in der Liebe bei Tarantino erscheint: als verändernder Einbruch in eine bestehende Form der Ordnung. Als ereignishafter Ausbruch sind die Szenen der Gewalt bei Kitano daher zugleich von äußerster Kürze. Der Opulenz, die Tarantino im sehr amerikanischen "Showdown at the House of Blue Leaves" an den Tag legt, begegnet Kitano mit einem Purismus der Gewalt, der fast schon ans Asketische grenzt. Den dramatischen Höhepunkt des Films, das Aufeinandertreffen Zatoichis und des von der gegnerischen Bande gekauften Samurais Hattori, zeigt Kitano, wie in den meisten seiner Filme Hauptdarsteller und Regisseur in einem, als einen streng ritualisierten Austausch weniger Gesten. Die Gestenhaftigkeit, die Kitanos Filme wiederum von der Dialogkunst Tarantinos unterscheidet, erfüllt sich in einem einzigen Schlag, den die Kämpfer ausführen und bei dem Zatoichi zwar am Hals verletzt, sein Gegenüber aber getötet wird.

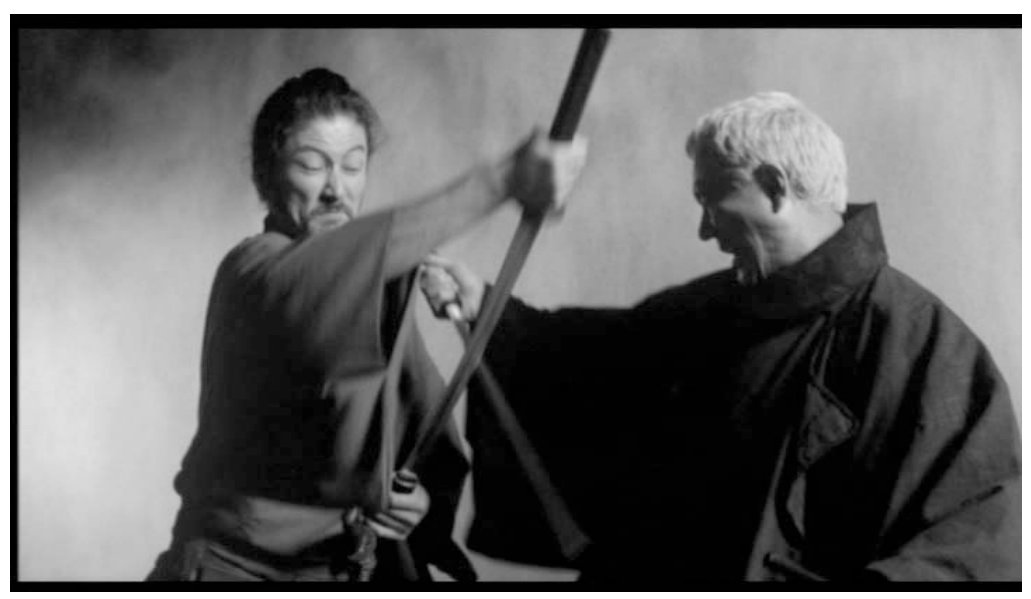

Abb. 3: Zatoichi kämpft gegen Hattori 
Die im Vergleich zu KILL BILL fast asketische Darstellung der Kampfszenen bestätigt, was Kitanos Film zum darstellerischen Prinzip seiner meisten Filme gemacht hat: eine Lakonie des Ausdrucks, die bisweilen komische, bisweilen tragische Züge trägt.

Das zeigt sich auch in Kitanos erstem in Amerika produzierten Film mit dem Titel BROTHER. Dem Titel entsprechend erzählt BROTHER die Geschichte, die den japanischen Yakuza Aikini mit drei unterschiedlichen >Brüdern` verbindet: dem Yakuzafreund, der von seiner neuen Familie dazu gezwungen werden soll, Aikini zu töten und ihm stattdessen den Ausweg nach Amerika zeigt, dem jüngeren leiblichen Bruder, den er in Los Angeles findet und mit dem zusammen er in kürzester Zeit eine äußerst lukrative Yakuzafamilie nach japanischem Muster errichtet, sowie dem schwarzen Freund des Bruders, dem er zu Beginn des Films mit einer zerbrochenen Flasche das Auge zerschneidet, um ihn am Ende mit dem verbliebenen Geld der Familie vor dem Zugriff der italienischen Mafia zu retten. BROTHER erzählt von der Solidarität, die Freundschaften kennzeichnet und die Stück für Stück durch Verrat verloren geht, und zugleich von der Begegnung Japans und Amerikas im Zeichen der Gewalt. Die Bereitschaft, für die eigene Ehre zu sterben, mit der der Film endet, zitiert Stereotypen des amerikanischen Gangsterfilms, etwa Brian de Palmas ScARfACE. Aikini, der einen italienischen Mafiaboss gekidnappt hat, wartet nach dessen Freilassung in einem amerikanischen Diner auf seine Hinrichtung. Als er vor die bald schon zersiebte Tür tritt, erwartet ihn eine Maschinengewehrsalve, die ihm jenen Tod schenkt, dem er schon in Japan überantwortet war und den er nun in Amerika findet.

Kitanos Filme verbindet mit Tarantinos Kino eine betonte >coolness $<$, die sich sowohl auf die Personen als auch auf die Erzählweise erstreckt. Die wortlose Gestenhaftigkeit, die Kitano durch eine äußerste Langsamkeit des Schnitts verstärkt, verhält sich zu den filmischen Darstellungsprinzipien Tarantinos jedoch geradezu spiegelverkehrt. Auf das ästhetische Prinzip der >overreaction`, das RESERVOIR DOGS, PULP FICTION wie KILL BILL kennzeichnet und das noch in den ungleich langsameren JACKIE BROWN Eingang gefunden hat, reagiert Kitano mit dem darstellerischen Mittel der Verknappung, um einer Sprachlosigkeit Raum zu geben, die den Mythos des einsamen Helden umrahmt, dekonstruiert und zugleich wieder einsetzt. Das Spiel von Dekonstruktion und Remythisierung, das Kitanos Kino begleitet, lässt sich auch an KILL BILL verfolgen, dessen zweiter Teil den Mythos, den das Schwert im ersten Teil begründet hat, in der Verdoppelung auflöst, um zugleich zu einem ganz anderen neuen Mythos zu finden. 


\section{Liebe}

Kitano lässt seinen Helden in BROTHER am Mythos Amerikas scheitern. Die Brutalität, mit der Aikini seinen Feldzug durchgeführt hat, um sich eine Machtposition innerhalb der Mafia zu erarbeiten, erweist sich als verzögerte Erfüllung des Todes, dem er in Japan überantwortet war. Die kulturellen Muster Japans und Amerikas bestimmen auch KILL BILL. Sie werden in den beiden Teilen des Films jedoch unterschiedlich akzentuiert. In KILl BILL: Volume 2 ist die in Japan eingesetzte Herrschaft des Schwertes, die Kill Bill: Volume 1 im Kapitel »The man from Okinawa« zeigt, gebrochen. Zwar reist Beatrix Kiddo mit dem Schwert durch die USA und Mexiko. Niemand aber stirbt mehr durch das Schwert: Weder Bills Bruder Budd, den Elle Driver durch das ihr eigene Gift der Schlange umbringt, noch Elle Driver selbst, der von Kiddo das zweite Auge herausgerissen wird und die aller Wahrscheinlichkeit am eigenen Gift stirbt, noch - und darauf kommt es hier an - Bill. Mit dem vielversprechenden Titel »Face to Face« konterkariert das letzte Kapitel des zweiten Teils den martialischen Höhepunkt des Showdowns, mit dem der erste Teil endete. Ein »Face to Face« vollzieht das Schlusskapitel auf ganz andere Weise als im Zweikampf der Braut mit O-Ren. Keine 88 Yakuzas sind mehr nötig, um Kiddo den Weg zu Bill zu bahnen - nur durch den Schlaf der eigenen Tochter muss sie hindurch. Das »Face to Face«, das der Schluss zeigt, steht nicht mehr im Zeichen des Schwertes, sondern dem der Wahrheitsfindung im und durch das Gespräch. Hatte Freud seine Patienten auf die Couch verwiesen, um durch die Technik der freien Assoziation zur Wahrheit zu gelangen, so vertritt Bill einen weitaus pragmatischeren Zugang. Da er Kiddo nicht traut und sie ihm nicht vertrauen kann, wie er selbst weiß, injiziert er ihr ein Wahrheitsserum mit dem Namen »The Undisputed Truth $\aleph^{35}$, um ihr eine Frage zu stellen, die nach mehr als drei Stunden Film und unzähligen Leichen seltsam banal und zugleich bekannt daherkommt: »Why did you run away from me with that baby? ${ }^{36}$ Von Angesicht zu Angesicht: An die Stelle des angekündigten Schwerkampfes - »I guess the idea is that we're crossing Hanzo swords $\aleph^{37}$ - tritt die, allerdings wiederum mit Mitteln der Gewalt, erzwungene Aussprache eines Expaares, das sich gegenseitig Rechenschaft gibt, warum die Beziehung nicht funktioniert hat: Zunächst Kiddo, indem sie ihren Entschluss der Flucht vor Bill aus der für sie neuen Mutterrolle begründet, schließlich Bill, indem er alkoholisiert zugibt, er habe `overreacted`, weil Kiddo ihm

35 KILL BILL: VOLUME 2, 01:39:39.

36 KILL BILL: VOLUME 2, 01:44:39.

37 KILL BILL: Volume 2, 01:47:27. 
das Herz gebrochen habe. Was im Mittelpunkt der Schlussszene steht, ist der Ausdruck der wechselseitigen Enttäuschung der beiden ehemaligen Partner über das Verhalten des anderen - eine Szene, die komisch anmuten könnte, würde sie nicht getragen von der Aussicht auf den finalen Schwertkampf, der jedoch ein allzu frühes und unvorgesehenes Ende nimmt: Nach der einleitenden Kampfesaufforderung "You and I have unfinished business $\aleph^{38}$, mit der die Braut schon O-Ren anrief, entwaffnet Bill Kiddo mit seinem Schwert, die wiederum seinen Hieb pariert, indem sie das Schwert mit der Scheide auffängt, um ihn darauf hin mit der >Five-Point-Palm-Exploding-Heart-Technique $<$ zu töten, ihm - in diesem Fall wörtlich - das Herz zu brechen. Liebe als Schwertkampf: Starb O-Ren Ishii im ersten Teil des Films durch das Schwert Hattori Hanzos, das die bereits tot geglaubte Braut wieder auf die Beine brachte, so endet Bill nicht durch das Schwert, sondern am gebrochenen Herzen - unwirklicher und theatralischer könnte der Film nicht enden. Die Frau steht als Siegerin da, weil sie den Hieb der Liebe parieren kann und in der Tochter neue Erfüllung findet. »Der Mann ist für das Weib ein Mittel: der Zweck ist immer das Kind « ${ }^{39}$, so lautet die geschlechterpolitisch eindeutige Formel in Nietzsches Zarathustra, die Bill, eben so einfach gestrickt wie Zarathustras Predigt über das Weib - »I'm the man ${ }^{40}{ }^{40}$ - in seinem Enthusiasmus für den `Superman` zu bestätigen scheint. ${ }^{41}$ Der Schluss, der Bills verletzte Männlichkeit überdeutlich zur Schau stellt, dreht den Verlauf des gesamten Films um und erlaubt es zugleich, ihn rückblickend neu zu lesen: Nicht mehr die Genugtuung der Rache steht im Zentrum des Films, sondern der Kampf der Mutter um ihr Junges, der in dem Schlussbild »The lioness has rejoined her cub and all is right in the jungle ${ }^{42}$ ironisch aufgenommen wird. Die Mythisierung des Schwertes, die der Film in der Tradition der Italo-Western und der Martial-Arts-Filme vollzieht, setzt der Schluss des Films außer Kraft, indem er die Geschichte der Rache in die Geschichte enttäuschter Liebe übergehen lässt, die zugleich in der Eintracht der neuen Familie aufgehoben wird, in der für den Mann kein Platz mehr ist. In der Dekonstruktion des Mythos, den der erste Teil erzählt, errichtet der zweite Teil einen neuen Mythos um eine Welt nach Bill, eine Postbillwelt, die das hysterische Weinen und Lachen der Braut auf der Toilette des Hotelzimmers einläutet, in das sie sich mit der Tochter zurückgezogen hat.

38 KILL BILL: VOLUME 1, 01:10:39.

39 Friedrich Nietzsche: "Also sprach Zarathustra", in: Sämtliche Werke. Kritische Studienausgabe, hrsg. von Giorgio Colli/Mazzino Montinari, München: de Gruyter 1980, S. 85.

40 KILL BILL: Volume 2, 00:06:29.

$41 \mathrm{Zu}$ Superman vgl. Umberto Eco: »The myth of Superman«, in: Diacritics (1972), S. 14-22.

42 KILL BILL: Volume 2, 01:58:48. 
Nach dem Schmerz also die Liebe, nach dem Mythos des phallischen Schwertes und dessen Dekonstruktion die Remythisierung mütterlicher Liebe, die die Macht der Differenzen anhält, um schließlich selbst der Dekonstruktion als einer Kritik anheim zu fallen, die der Film selbst nicht mehr leistet - es sei denn, Beatrix Kiddo fiele durch die Hand der Tochter von Vernita Green, besser noch durch die der eigenen Tochter, die die Mutter wie einen Goldfisch auf dem Teppich zappeln ließe. Die mythische Kraft unerzählter Geschichten scheint ebenso groß zu sein wie die der erzählten. Es reicht bereits, wenn die Übertragung von Schmerz in Affekte angedeutet wird, um jenes Quantum Lust frei zu setzen, auf dem die Identifikation aufsetzen kann - eine Identifikation, vor der allein die Rückübersetzung der Affekte in die Schmerzquantitäten schützen kann, in denen Freud den Ursprung des Lebens erkennen wollte, den Urknall, der aus unbelebter Materie eine reizbare, lebendige, eben: schmerzfähige Substanz machte: Bang Bang.

\section{Silly Caucasian Boy}

>Silly Caucasian boy likes to play with samurai films`, so ließe sich Tarantinos eklektizistischer Umgang mit der Tradition von Western und Eastern kritisch zusammenfassen. Die Fülle an Zitaten und Verweisen, die der Film aufweist, ist sicherlich ebenso wenig Garant von Qualität wie die Anzahl intertextueller Verweise in literarischen Texten. ${ }^{43}$ Was sie beweisen, ist allein die Tatsache, dass sich auch Tarantinos Kino in die ironische Geschichte der Postmoderne einreihen lässt, der zufolge Texte unablässig auf andere, ihnen vorgängige Texte verweisen und eine Geschichte sich auch im Film nicht mehr anders erzählen lässt als durch den Rückverweis auf andere Filme und Texte. Insofern scheint das Medium des Films trotz aller Unkenrufe vom Ende der GutenbergGalaxie von dem des Buches nicht wesentlich unterschieden zu sein: Intertextualität, Intermedialität und Interkulturalität sind Begriffe, die beiden Medien im Rahmen kulturwissenschaftlicher Analysen gleichermaßen zustehen. Was in KILL BILL auf dem Spiel steht, ist jedoch zugleich etwas anderes: eine Affektpolitik, die sich nicht einfach durch den Verweis auf die angebliche Trivialität des Films abtun lässt. PULP

43 Dass Tarantino die Verweistechnik, die seine Filme kennzeichnet, auf die Spitze getrieben hat, unterstreicht schon Robert Fischer: »Aber keiner der früheren Filme hat das Spiel mit Bezügen, Verweisen und Entlehnungen so konsequent und so weit betrieben wie KILL BILL, keiner hat in ähnlichem Maß darauf verzichtet, irgend etwas anderes sein zu wollen als pure, reine Hommage.« Robert Fischer: »Kill Bill«, in: Robert Fischer/Peter Körte/Georg Seeßlen: Quentin Tarantino, S. 198-234, hier S. 216. 
FICTION hatte die Trivialität des Genres schon im Titel mitreflektiert: >Pulp is fiction` wäre die Folgerung, der zufolge aus der Übertragung von Übertragungen, die zeitgenössischen Theorien zufolge Medien sind, eben auch nichts anderes als weiterer Übertragungsschrott entstehen kann. Ihn neu aufzubereiten, um aus alten Gefühlen eine Erlösungssuppe zu köcheln, wie es THE MATRIX in der Geschichte von Trinity und PASSION gleich im Rückverweis auf die einzige Geschichte vorführt, die bis heute für die Utopie vom Ende aller Leiden einsteht, scheint nur ein Weg zu sein, der dem zeitgenössischen Kino offen steht. »Heiße Medien verlangen daher nur in geringem Maße persönliche Beteiligung, aber kühle Medien in hohem Grade persönliche Beteiligung oder Vervollständigung durch das Publikum « ${ }^{44}$, schreibt McLuhan die Differenz zwischen heißen Medien wie dem Film und kalten Medien wie dem Fernsehen oder der Schrift fest. Die Schrift der Moderne, von sich aus bereits ein kaltes Medium, zeichnet sich, wie Flaubert und Kafka zeigen, durch einen Entzug der Affekte aus, die zu neuen Verhaltenslehren der Kälte geführt haben. ${ }^{45}$ >Coolness $<$ ist eines der Attribute, das Tarantinos Figuren nicht anders als die Kitanos unbestritten für sich in Anspruch nehmen können. ${ }^{46}$ Was Kitano und Tarantino hinter der betonten >coolness ihrer Figuren und ihres Erzählstils sichtbar machen, ist die mediale Überhitzung der sie verbindenden Genres $^{47}$, deren hybride Kreuzung neue Intensitäten schafft. Anders als in PASSION oder THE MATRIX aber stellt Tarantino das Stigma des Schmerzes, von dem KILL BILL erzählt, nicht in den Kontext religiöser Erlösungsphantasien - auch nicht, wenn das blutbesudelte Gesicht der Braut, mit dem der Film beginnt, manches aus der Tradition der Schmerzensmutter aufnehmen mag. Wie schon in PULP FICTION ist es nicht die hybride Kreuzung unterschiedlicher Genres allein, die Tarantinos Filmen ein eigenes Gewicht gibt. Tritt der starke Affekt des Zorns in den Mittelpunkt der Geschichte, so öffnet sich hinter ihm zugleich eine Liebesgeschichte, die sich in ihrer Schlichtheit nur indirekt erzählen lässt, indem sie Affekte nicht mehr unmittelbar in Szene setzt, sondern nur noch auf den Umwegen der Ironie. Die Rachegeschichte, die Tarantinos KILL BILL zu erzählen scheint, ist ein solcher Umweg, der, hilflos genug, aus Trabanten des Todes Lebenswächter zu machen versucht, indem er noch aus dem

44 Marshall McLuhan: Die magischen Kanäle. Understanding Media, Frankfurt a.M.: Fischer 1970, S. 32.

45 Vgl. Helmut Lethen: Verhaltenslehren der Kälte. Lebensversuche zwischen den Kriegen, Frankfurt a.M.: Suhrkamp 1994.

46 Paul A. Woods erkennt in Tarantino daher »the very epitome of cool. «Paul A. Woods: King Pulp. The Wild World of Quentin Tarantino, London: Plexus 1998, S. 72.

47 Zum Thema Genre vgl. die Analyse von PulP FICTION von Knut Hickethier, in: Jürgen Felix (Hg.): Moderne Film Theorie, Mainz: Bender 2002, S. 97-103. 
>Lonely Grave of Paula Schultz` den Weg ans Licht dieser Welt weist und die zerstörerische Reise der Braut einem Ziel unterstellt, das sie im Verblendungszusammenhang der Rache nicht kennt, bis sie es erreicht hat - unwahrscheinlich genug, zugleich aber der exzentrischen Bahnen des Pathos folgend, von der schon Hölderlin in seinen Ödipus-Anmerkungen berichtete, dass sie »den Menschen seiner Lebenssphäre, dem Mittelpuncte seines inneren Lebens in eine andere Welt entrükt und in die exzentriche Sphäre der Todten reißt. $^{48}$

\section{Literaturverzeichnis}

Aristoteles: Poetik, Stuttgart: Reclam 1982.

Aristoteles: Rhetorik. Übersetzt, mit einer Bibliographie, Erläuterungen und einem Nachwort von Franz G. Sieveke, München: Fink 1980.

Doris Bachmann-Medick (Hg.): Kultur als Text. Die anthropologische

Wende in der Literaturwissenschaft, Frankfurt a.M.: Fischer 1996.

Eco, Umberto: »The myth of Superman«, in: Diacritics (1972), S. 14-22.

Fischer, Robert/Körte, Peter/Seeßlen, Georg: Quentin Tarantino, Berlin:

Bertz + Fischer 2004 (film: 1).

Fischer, Robert: »Kill Bill«, in: Robert Fischer/Peter Körte/Georg

Seeßlen: Quentin Tarantino, Berlin: Bertz + Fischer 2004 (film: 1), S. 197-234.

Freud, Sigmund: Studien zur Hysterie, in: Gesammelte Werke I, Frankfurt a.M.: Fischer1999, S. 312.

Hickethier, Knut: »Pulp Fiction. USA 1994; Regie: Quentin Tarantino«, in: Jürgen Felix (Hg.): Moderne Film Theorie, Mainz: Bender 2002, S. 97-103.

Hölderlin, Friedrich: »Anmerkungen zum Ödipus«, in: Sämtliche Werke, Briefe und Dokumente, hrsg. von D. E. Sattler, Bremer Ausgabe. Band X. 1802-1804, München: Luchterhand 2004.

Hölderlin, Friedrich: Sämtliche Werke, Briefe und Dokumente. Hrsg. von D. E. Sattler, Bremer Ausgabe, Band VI. 1797-1799, München: Luchterhand 2004.

Holm, D. K.: Kill Bill. An Unofficial Casebook, London: Glitterbooks 2004.

Homer: Ilias, griechisch und deutsch. Übertr. von Hans Rupé, Düsseldorf [u.a.]: Artemis \& Winkler 2001.

48 Friedrich Hölderlin: Anmerkungen zum Ödipus, in: Sämtliche Werke, Briefe und Dokumente, hrsg. von D. E. Sattler, Bremer Ausgabe. Band X. 1802-1804, München: Luchterhand 2004, S. 156. 
Lacan, Jacques: »Le stade du miroir comme fomateur de la fonction du Je«, in: Écrits, Paris: Seuil 1966, S. 93-100.

Körte, Peter: »Geheimnisse des Tarantinoversums. Manie, Manierismus und das Quäntchen Quentin - Wege vom Videoladen zum Weltruhm«, in: Robert Fischer/Peter Körte/Georg Seeßlen: Quentin Tarantino, Berlin: Bertz + Fischer 2004 (film: 1), S. 11-64.

Koppenfels, Martin von: »Schmerz. Lessing, Duras und die Grenzen der Empathie«, in: Robert Stockhammer: Grenzwerte des Ästhetischen, Frankfurt a.M.: Suhrkamp 2002, S. 118-145.

Kosěnina, Alexander: »Verstellung«, in: Historisches Wörterbuch der Philosophie, hrsg. von Joachim Ritter, Karlfried Gründer und Gottfried Gabriel. Band 11: U-V, Darmstadt: Wissenschaftliche Buchgesellschaft 2001, S. 938-942.

Lethen, Helmut: Verhaltenslehren der Kälte. Lebensversuche zwischen den Kriegen, Frankfurt a.M.: Suhrkamp 1994.

Luhmann, Niklas: Liebe als Passion. Zur Codierung von Intimität, Frankurt a.M.: Suhrkamp 1982.

Marshall McLuhan: Die magischen Kanäle. Understanding Media, Frankfurt a.M.: Fischer 1970.

Friedrich Nietzsche: »Also sprach Zarathustra«, in: Sämtliche Werke. Kritische Studienausgabe, hrsg. von Giorgio Colli/Mazzino Montinari, München: de Gruyter 1980.

Seeßlen, Georg: »Zärtliche Zerstörungen. Anmerkungen zur Musik in Tarantinos Filmen«, in: Robert Fischer/Peter Körte/Georg Seeßlen: Quentin Tarantino, Berlin: Bertz + Fischer 2004 (film: 1), S. 65-86.

Woods, Paul A.: King Pulp. The Wild World of Quentin Tarantino, London: Plexus 1998. 
